\title{
Issues related with the analysis of nanomaterials
}

\section{Christine Vauthier}

Institut Galien Paris Sud

UMR CNRS 8612, Université Paris-Sud

5 Rue J.B. Clément

92296 Chatenay-Malabry Cedex, France

Published in: "Handbook of nanomaterials in analytical chemistry", Editor Chaudhery Mustansar Hussain, Publisher, Elsevier, Amsterdam, The Netherlands, 2020. Chapter 19, pp. 473-790. DOI: 10.1016/B-978-0-12-816699-4.00019-0. ISBN print: 978-0-12-816699-4 ISBS Ebook: 978-0-12-8172902.

Corresponding author

Institut Galien Paris Sud

UMR CNRS 8612, Université Paris-Sud

5 Rue J.B. Clément

92296 Chatenay-Malabry Cedex, France

Email: Christine.vauthier@u-psud.fr

Phone +33146835603 


\section{Content}

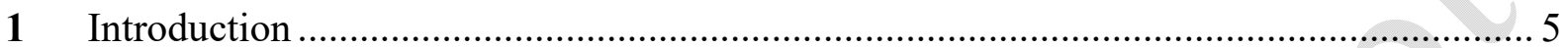

2 Nanomaterial characteristics: identifying key attributes ........................................... 7

3 Analyzing nanomaterial physical attributes with automated and standardized methods 10

3.1 Standardization of methods for the analysis of nanomaterials ................................ 10

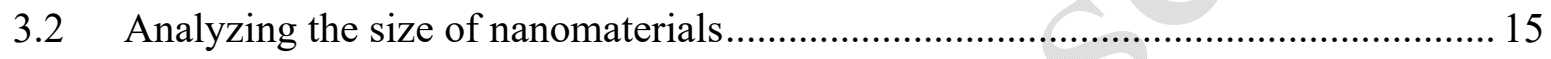

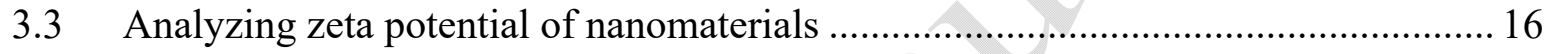

$4 \quad$ Analyzing attributes of nanomaterials using other methods...................................... 17

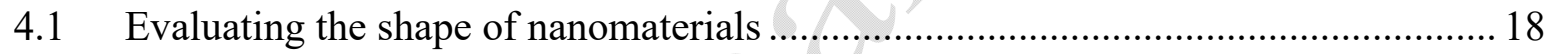

4.2 Evaluating the porosity and crystallinity with general methods for the characterization

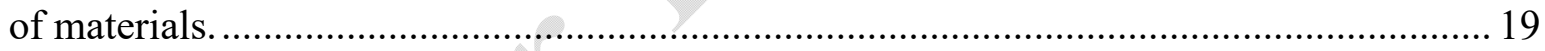

4.3 Analysis of the intimate structure of nanomaterials ........................................ 20

4.4 Thorough analysis of nanomaterial surface attributes ........................................ 21

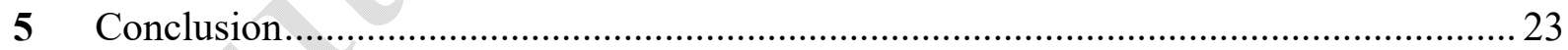

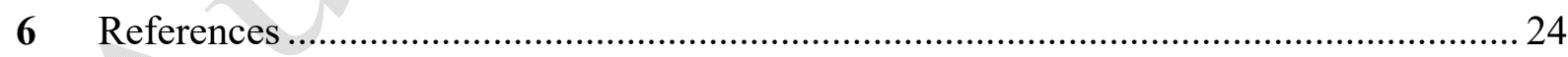




\section{List of abbreviations}

AFM: Atomic Force Microscopy

Cryo-TEM: Cryo-Transmission Electron Microscopy

DLS: Dynamic Light Scattering

ELS: Electrophoresis Light Scattering

ELS-PALS: Electrophoresis Light Scattering coupled with Phase Analysis Light Scattering

EU-NCL: European Nanomedicine Characterization Laboratory

FDA: Food and Drug Administration in the United States of America

ICH: International Council on Harmonization of Technical Requirements for Registration of Pharmaceuticals for Human Use

ISO International Standardization Organization

NIH-NCL: National Institute of Health - Nanomedicine Characterization Laboratory

NIST: National Institute of Standard and Technology

NTA: Nanoparticle Tracking Analysis

SEM: Scanning Electron Microscopy

TEM: Transmission Electron Microscopy

TRPS: Tunable Resistive Pulse Sensing

XPS: X-Ray Photo-Electron Spectroscopy 


\begin{abstract}
Objects which external size in at least one dimension is below $100 \mathrm{~nm}$ are nanomaterials. Such materials display unique properties compared with bulk with same composition. They interest many fields of the industry including applications in medicines. Properties, functionality and activity depend on their chemical nature and physical attributes. This chapter was focused on the analysis of their physical attributes considering (i) the different attributes interested to analyse to define nanomaterial identity and insure quality in an industrial perspective, (ii) methods of analysis for which automated measurement instruments and standardization were developed, (iii) needs to analysis of other key attributes. A wide range of physical attributes can define nanomaterials. Only the size, size distribution and surface charges can be evaluated with automated instruments and were the subject of a standardization. Additional methods and standard materials are needed to improve quality control of nanomaterials.
\end{abstract}

\title{
Key words:
}

Quality control, ISO standard, size, zeta potential, shape, key attributes, nanomaterial surface, nanomaterial structure, safety, activity

\section{Words suggested for the index:}

Standard, size, polydispersity, zeta potential, shape, porosity, crystallinity, surface, nanomedicines, safety, quality control, structure, dynamic light scattering, Electrophoretic light scattering, tunable resistive pulse sensing, nanoparticle tracking analysis, field flow fractionation, centrifugation, X-ray photo-electron spectroscopy, Atomic force microscopy, transmission electron microscopy, scanning electron microscopy. 


\section{Introduction}

Nanomaterials comprise objects which external size in at least one dimension is below $100 \mathrm{~nm}$ (references are given under "Nanomaterial definition"). They are materials built from the assembly of many molecules that can be identical or of different nature. Because of their very small size, highly developed specific surface area is the seat for an intense interfacial reactivity which is not found in the same materials occurring at a larger size that exposed a much smaller specific surface area to the surrounding media. Thanks to their very small size, nanomaterials display unique properties which differ from those found on material of the same composition occurring in bulk. They interest many fields of the industry (Thirumavalavan et al., 2016, Singer et al., 2018), in analytical science (Hussain, 2018) and are considered for applications in medicines (Mura and Couvreur, 2016, Juliano, 2016, Pelaz et al., 2017, Ventola et al., 2017, Lamch, 2018)

Properties, functionality and activity of nanomaterials closely depend on their chemical nature and physical attributes. As for all manufactured products, quality and safety of use require strict quality control analysis. The chemical composition can be accessed with analytical techniques generally used to analyze other materials. In contrast, analysis of physical attributes of nanomaterials is more difficult to achieve due to the small size and requires the development of specific analytical methods. In addition, nanomaterials generally occur as a population of individual nano-objects. In the ideal case, the population is composed of perfectly identical nano-objects. However, very few engineering methods are suitable to produce such "ideal" nanomaterials. In general, they are composed of individual nano-objects each having their own characteristics that can differ more or less from that of the mean value found for the population. Deviation from the mean value of key attributes may significantly influence the performance 
of the nano-object in the framework of its intended use. So, the distribution of the population around the mean value of key attributes is an important characteristic to determine too. In this context, it is noteworthy that even accurate evaluations of the size and size distribution of a nanomaterial which are obvious attributes to determine are challenging tasks (Anderson et al., 2013, Crist et al., 2013, Clogston et al., 2016, Varenne et al., 2016). Artifacts may also contribute to the misinterpretation of the measurement results hence to draw incorrect conclusions with possible impact evaluating possible hazards of nanomaterials (Pertersen et al., 2014).

At present, very few nanomaterial attributes can be evaluated in routine. These include general attributes such as the size, size distribution and surface charges through the evaluation of their zeta potential. Several affordable instruments to achieve their measurements are available on the market. Morphology which is another general attribute of nanomaterials can be determined by electron microscopy or using atomic force microscopy (AFM). These techniques provide images of nanomaterials and can be found on technological platforms in Universities and in some private analytical laboratories. Attributes that are more closely linked with functionalities exploited in the application of the nanomaterial are more challenging to evaluate. Their determination is often pending to the application of analytical methods used in research. Today, the low number of methods of characterization of nanomaterials that can be applied in routine considerably limits the number of attributes evaluated during quality control assessment of nanomaterials. This is an issue for the development of such materials and especially for nanomedicines which safety and activity cannot be evaluated confidently only from their measurable general attributes as pointed out in several recent papers (Hamad et al., 2010, Dobrovolskaia et al., 2016, Anchordoquy et al., 2017, Siegrist et al., 2018, Coty and Vauthier, 2018). The objective of this chapter was to review and discuss the present state of the art on the analysis of physical attributes of nanomaterials. A first part attends to list the different attributes 
that may be used to characterize nanomaterials in relation with their intended applications. The second part points out the few attributes that can be determined in routine thanks to the existence of suitable marketed measurement instruments. This part of the chapter will also examine the stage of the standardization and remaining analytical issues. In the last part, the evaluation of other attributes will be considered based on techniques applied in research or methods that are used to characterize non-nanomaterials. From a few examples, problems that may arise using techniques not-specifically designed for the characterization of nanomaterials will be discussed. While this chapter attempts to discuss issues related with the analysis of nanomaterials in general, specific examples were drawn from the characterization of nanomedicines designed for a use in human clinics.

\section{Nanomaterial characteristics: identifying key attributes}

Nanomaterials can be described by different physical attributes. The size is the more obvious parameter. It is generally systematically included within the set of characterization performed in quality control. The size distribution as well as the morphology are other parameters that are evaluated during development stages of products and that can be included in quality control as a mean to check the reproducibility of the manufacturing process. Depending on the application, nanomaterials are designed to achieve a well define function which depends on specific attributes. Those for which variations beyond certain limits can compromise the functionality of the nanomaterial are identified as key attributes and carefully considered in procedures dedicated to insure the quality and safety of the product. Key attributes are defined on a case by case basis for each nanomaterial consistently with the intended use.

Applications in the domain of medicine include a wide range of nanomaterials developed for therapeutic and/or diagnostic purposes (Zhang et al., 2012, Thirumavalavan et al., 2016, Singer 
et al., 2018). Their use can differ from their composition and structures a lot depending on the specific application they are designed for (Kamarly et al., 2012, Thirumavalavan et al., 2016). Several nanomedicines are being used in vivo and their hazard evaluation is an issue for their translation into the clinic. Although, characterizations are generally closely related with the intended use, as pharmaceutics or medical devices, the product needs to comply with stringent legislative frameworks (see for instance Lakkireddy and Bazile, 2016). Different aspects are taken into consideration including the characterization of their physicochemical attributes with specific requirements due to the small size of nano-abjects composing nanomedicines. Characterizing nanomaterials entering the composition of nanomedicines remains challenging on different levels (Crist et al., 2013, Clogston et al., 2016, Siegrist et al., 2018). At first, there is a tremendous number of ways by which nanomedicines can be used as a nanomedicine. For each application there is a need to elucidate how the nanomedicine is interacting with the biological environment to achieve an optimal control of their activity identifying corresponding key attributes of the nanomaterial. Then, our present knowledge for a safe evaluation and supervision of nanomedicines remains partial. Critical attributes which control the pharmacological activity in vivo are not fully elucidated even in the more general cases considering nanomedicines designed to be administered by the oral or intravenous routes (Dobrovolskaia 2016, Lakkireddy et al., 2016). This hampers the definition of a set of relevant key attributes for the given type of applications. Besides, nanomedicines occurred in a wide range of compositions and structures including quite complex structures that improve the difficulty to establish general rules to perform their characterization (Kamaly et al., 2012). Finally, methods are still cruelly missing to evaluate several of the key attributes of nanomedicines that would be worth to determine in routine (Brenner-Hoffmann et al., 2018). In this context, a consensus was established identifying a minimum of physical attributes that need to be determined for a nanomedicine which includes the size and size distribution (Gloria 
et al., 2018). In addition to size characteristics, the morphology and surface charge of the nanomaterials are generally evaluated too as these attributes are also known to impact safety and clinical manifestations of many nanomedicines designed to be administered by the intravenous route (Siegrist et al. 2018). These are also attributes that influence the in vivo fate of nanomedicines delivered by a mucosal route including the oral route (Lakkireddy et al., 2016). Other characteristics may be required but their determination is generally demanding and may even be problematic as there is no existing easy method to perform the analysis. For instance, a precise characterization of the surface of nanomedicines intended to be used by the intravenous route would be justified because pharmacology and toxicity of the products is closely controlled by subtle characteristics of the nanoparticle surface including the density and conformation of the chains of hydrophilic macromolecules grafted on the surface of the nanoparticle core (Agrawal et al., 2009, Hamad et al., 2009, Coty et al., 2017, Coty and Vauthier, 2018). Consistently, parameters such as the surface coating coverage, the topology, and the deformability were included in the list of parameters that may be of interest to consider while developing nanomedicine in a document proposed by the Food and Drug Administration of the United States of the America (FDA) (See Cruz et al., 2013). However, these parameters are rarely determined as suitable methods for their determinations are not available. The characterization of nanomedicine should also include an evaluation of the stability of the dispersion (Siegrist et al., 2018, Gloria et al., 2018). The stability can be considered on the chemical view point but must also evaluate any possible changes occurring in the structure of the nanomaterial and at the level of the dispersion. As already mentioned, the chemical nature of the nanomedicines can be evaluated through general methods applied in analytical chemistry. Evaluation of the stability of the dispersion can be performed measuring the size of the particles and evaluating the size distribution at different times upon storage. Changes in these characteristics would suggest that the colloidal stability of the dispersion was affected during 
storage. An increase in the size and size distribution would indicate an aggregation of the nanoparticles while a decrease in the size would suggest a degradation of the particles. Deciphering the structure of a nanomaterial is demanding and requires very specific methods and the expertise of seasoned scientists. Demonstrating that the structure of the nanomaterial is not affected upon storage will be the most difficult task to achieve analyzing the stability of a nanomedicine. This aspect is eventually considered during early preclinical development stages of a nanomedicine. The following parts give an overview of the different methods applied for the analysis of several attributes of nanomaterials.

\section{Analyzing nanomaterial physical attributes with automated and standardized methods}

\subsection{Standardization of methods for the analysis of nanomaterials}

Standardization in the domain of nanomaterial is a young area. The result of a search with the term "nanomaterial" on the web site of the International Standardization Organization (ISO web site) has mentioned 40 documents defining terms and definitions and 82 standards including technical reports and specifications (ISO web site, 21/01/2019). Most standards are very recent. The number of the standards drop down to 61 while using the words: "nanomaterial", "method", "characterization" in the search engine of the ISO web site and very few were directly devoted to the determination of nanomaterial physical attributes (Table 1). 


\begin{tabular}{|c|c|}
\hline Ger & $\begin{array}{l}\text { ISO 5725-1:1994: Accuracy (trueness and precision) of measurement methods } \\
\text { and results-Part 1: General principles and definitions } \\
\text { ISO 5725-3:1994: Accuracy (trueness and precision) of measurements } \\
\text { methods and results-Part 3: Intermediate measures of the precision of a } \\
\text { standard measurement method. } \\
\text { ISO Guide 35:2006: Reference materials - General and statistical principles } \\
\text { for certification. } \\
\text { ISO/TS 12805:2011(en) Nanotechnologies - Materials specifications - } \\
\text { Guidance on specifying nano-objects } \\
\text { ISO/TR 13014:2012(en) Nanotechnologies - Guidance on physico-chemical } \\
\text { characterization of engineered nanoscale materials for toxicologic assessment } \\
\text { ISO/TR 13329:2012 Nanomaterials -- Preparation of material safety data sheet } \\
\text { (MSDS) } \\
\text { ISO/TS 20477:2017 Nanotechnologies -- Standard terms and their definition } \\
\text { for cellulose nanomaterial }\end{array}$ \\
\hline $\mathrm{Siz}$ & $\begin{array}{l}\text { ISO 13322-1:2004 Particle size analysis - Image analysis methods - Part 1: } \\
\text { Static image analysis, methods } \\
\text { ISO/DIS 19749(en) Nanotechnologies - Measurements of particle size and } \\
\text { shape distributions by scanning electron microscopy } \\
\text { ISO 22412:2017(en) Particle size analysis - Dynamic light scattering (DLS) } \\
\text { ISO 19430:2016(en) Particle size analysis - Particle tracking analysis (PTA) } \\
\text { method } \\
\text { ISO/TS 21362:2018(en) Nanotechnologies - Analysis of nano-objects using } \\
\text { asymmetrical-flow and centrifugal field-flow fractionation }\end{array}$ \\
\hline $\begin{array}{l}\text { Morpholo } \\
\text { Shape/ } \\
\text { Structure }\end{array}$ & $\begin{array}{l}\text { ISO/TS 10797:2012(en) Nanotechnologies - Characterization of single-wall } \\
\text { carbon nanotubes using transmission electron microscopy } \\
\text { ISO/TS 10798:2011(en) Nanotechnologies - Charaterization of single-wall } \\
\text { carbon nanotubes using scanning electron microscopy and energy dispersive } \\
\text { X-ray spectrometry analysis }\end{array}$ \\
\hline & $\begin{array}{l}\text { ISO 13099-1:2012(E): Colloidal systems-Methods for zeta-potential } \\
\text { determination-Part 1: Electroacoustic and electrokinetic phenomena. } \\
\text { ISO 13099-2:2012(E): Colloidal systems-Methods for zeta-potential } \\
\text { determination-Part 2: Optical methods. } \\
\text { ISO 13099-3:2012(E): Colloidal systems-Methods for zeta-potential } \\
\text { determination-Part 3: Acoustic methods. } \\
\text { ISO/TS 14101:2012(en) Surface characterization of gold nanoparticles for } \\
\text { nanomaterial specific toxicity screening: FT-IR method } \\
\text { ISO/TR 19997:2018(en) Guidelines for good practices in zeta-potential } \\
\text { measurement }\end{array}$ \\
\hline
\end{tabular}

Table 1: ISO standards for the characterization of nanomaterials. TR technical report. TS

Technical specification 
Analyzing physical attributes of nanomaterials is pending to the existence of methods that have reached an enough degree of maturity making possible the development of a standardization. As for any kind of analysis, standards are needed to provide reliable measurements being trusted and accepted internationally. Then, applications of measurement methods are pending to the availability of affordable instruments. The nanometrology is a subfield of metrology that emerged to cover the science of measurements dedicated at the nanoscale. It was introduced to answer urgent needs for performing measurement at this size scale concomitantly with the expansion of industrial applications and processes involving nanomaterials that required evaluation methods to bring these nanotechnology-based products safely into the market place. Issues are remaining to develop standardized methods and standard operating protocols needed to produce reliable measurements with internationally recognized methods. Efforts in standardization are also needed to develop suitable reference materials, methods and corresponding instruments. Currently, very few standards have been established to achieve the characterization of nanomaterials (Mansfield et al., 2017, See table 1 for reference of ISO sandards). They were developed to achieve measurements of the size by scanning electron microscopy (SEM), dynamic light scattering (DLS), tunable resistive pule sensing (TRPS) particle tracking analysis (PTA), asymmetrical-flow centrifugal field-flow fractionation (AsFFFF); size distribution by SEM and zeta potential by acoustic and optical methods. These attributes can be determined with automated instruments. The morphology is another parameter for which a standard has been established using SEM but no automated procedures has been yet developed and analysis of images remains mostly manual and greatly depends on the quality of the images produced from the techniques. A general ISO standard explains methods for the analysis of images analyzing the size of particles (See table 1). The Nanomedicine Characterization Laboratory created by the National Institute of Health (NIH-NCL) in Frederik, MD, USA in the early 2000's was settled to support clinical development of nanomedicines 
with the mission to provide with resources and characterization services (NIH-NCL). It is a major player in the development of assay cascades and standard operating conditions for the characterization of physical attributes of nanomaterials composing nanomedicines. The NIHNCL have published several measurement protocols that are available on its web site (NIHNCL assay cascade). A similar service is provided by the European-Nanomedicine Characterization Laboratory (EU-NCL) built in partnership with the NIH-NCL in the 2010s' (EU-NCL, EU-NCL assay cascade). Different methods with corresponding automated measurement instruments can be used to determine the size and zeta potential of nanomaterials. The methods are generally indirect meaning that the mesurand is not the measured parameter. The mesurand is deduced from the measured parameter from the application of an appropriate model. Thus, values found for the evaluated attribute generally depends on the measurement method. For instance, most methods applied to determine the size of nanomaterial dispersed in an aqueous media provide with a hydrodynamic radius/diameter of the particles which corresponds to the diameter/radius of the particles with a layer of hydration (Figure 1A).
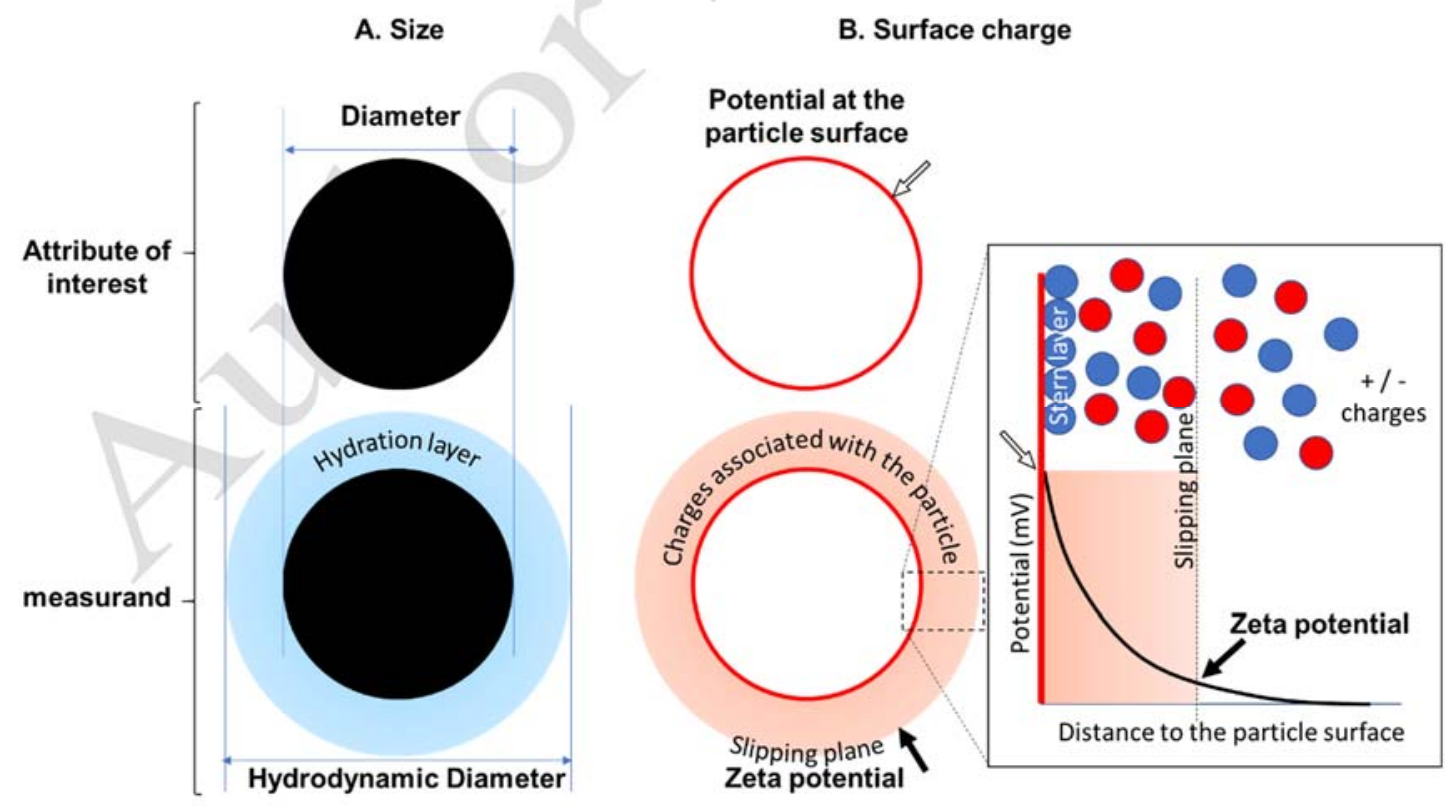

Figure 1. Attributes vs mesurands. (A) Size, (B) Surface charge. 
The hydrodynamic diameter is deduced from the diffusion coefficient of the particles which is the measured parameter of the Brownian motion. The mean size and size distribution provided from measurements performed by DLS methods are expressed based on the intensity of the signal collected by the photon detector. It may differ from that deduced from the TRPS method which evaluates the size of particles by measuring an electric pulse which intensity is proportional to the volume of electrolyte displaced by a single particle moving through a pore of calibrated volume assuming that the displaced volume of the electrolyte corresponds to that of the particle. With this latter method, the evaluation of the size distribution is provided in number. The PTA method also provides with a size distribution in number. As the different methods are given different information about the size and size distribution of nanomaterials, procedures intended to validate measurement protocols and to qualify measurement instruments should be done with standards certified for the method applied by the measurement instrument to be used. Several NIST traceable particle size standards with different sizes and chemical natures are available. Most of them are certified by electron microscopy which is a direct method for the determination of the size and only few are available with a DLS certification. Efforts are ongoing to extend the range of standards certified by the different size measurement methods. Regarding the validation of measurement protocols, procedures to perform such validation have not yet been described in ISO standards. However, procedures to validate protocols of size measurements by DLS and of evaluation of the zeta potential of nanomaterials by electrophoresis light scattering coupled with phase analysis light scattering (ELS-PALS) were published in the literature a few years ago (Varenne et al., 2015a, b, 2016a, In Press). The International Council on Harmonization of Technical Requirements for Registration of Pharmaceuticals for Human Use (ICH) was followed as much as possible to achieve these validations but some adaptations were needed to take into account that methods of 
characterization of nanomaterials did not require a calibration in contrast to the methods being the subject of this text. Robustness, precision and trueness of protocols were demonstrated using standard materials and following recommendations of the Guide to the expression of uncertainty in measurement (GUM 1995). Those included National Institute of Standard and Technology (NIST) standards of different sizes that were used to validate the proposed size measurement protocol and NIST standards of positive value and reference material of negative value that were used to validate the proposed protocol evaluating zeta potential. A procedure to transfer the validated protocols have also been reported (Varenne et al., 2017).

\subsection{Analyzing the size of nanomaterials}

Size of nanomaterials can be evaluated by different methods using automated instruments. The DLS is the most widely used method. First automated instruments appeared in the mid 1980s. Instruments working on new methodologies were introduced during the last 10 years. They are based on TRPS, NTA, centrifugation and field flow fractionation (FFF). The DLS method is a batch method meaning that it analyzes the dispersion in its all. TRPS and NTA are performing measurements on each particle composing the sample. Methods based on centrifugation and FFF separate particles according to their size prior to measure their size.

Analyzing the size of a nanomaterial remains challenging even with a well-established DLS method. This was revealed for instance in inter-laboratory comparisons (Langevin et al., 2018a). It also appears comparing size measurements performed with different DLS instruments (Varenne et al., 2016b, Langevin et al., 2018 b). Applying known precautions to perform measurements, results of size measurements of a sample of nanomaterial composed of nano-objects of homogenous size with narrow size distribution are generally accurate and precise (Varenne et al., 2015a, 2016a). In contrast, their application to the characterization of polydisperse nanomaterials is problematic. The intensity of the scattered light is dominated by the larger particles contained in the sample, the signal due to the smaller particles can be 
partially or totally hindered making difficult their detection. This is a well-known limitation of DLS methods. Improvements have been implemented in the youngest generation of instruments, but size measurements performed on a highly polydisperse dispersion remains problematic [Varenne et al., 2016]. More generally, results provided by DLS instruments may be different from those obtained from other measurement methods [Filipe et al., 2010, Varenne et al., 2016b, Anderson et al., 2013 Langevin et al., 2018 b]. Prior selecting DLS as sole technique to measure the size of a given nanomaterial, it is recommended to perform measurements with at least two methods working on different principles including one method based on particle by particle analysis (TRPS, NTA) or including a separation method (analytic centrifugation, field flow fractionation) (Varenne et al., 2016, Gloria et al., 2017, Langevin et al., $2018 \mathrm{~b}$ ). This shows that even the characterization of a very basic attribute such as the size of nanomaterials is complicated. It requires a thorough investigation based on the use of several techniques that are needed to perform an accurate evaluation of the polydispersity of the sample and obtain relevant information about the size of the nano-objects composing a sample.

\subsection{Analyzing zeta potential of nanomaterials}

The second attribute of nanomaterials for which standards have been established is the determination of the zeta potential (see table 1 for ISO standard reference). Very few reference materials are available yet. They were used for he validation of a measurement protocol by ELS-PALS (Varenne et al., 2015b). The zeta potential gives indications about the surface charge of nanomaterial. The charge at the surface of the nanomaterial is a difficult parameter to evaluate in contrast to the zeta potential that corresponds to the charges at the slipping plan between charges associated with the particles and charges from the bulk of the dispersion medium (Figure 1B). The value greatly depends on the nature of the dispersing medium and concentration in electrolytes. This information is needed while giving the result. The easiest methods evaluating the zeta potential of a nanomaterial are indirect based on the measurement 
of the electrophoretic mobility of the particles. Then, the zeta potential is calculated from this parameter using appropriate models. Affordable instruments are available to evaluate zeta potential of nanomaterials which is the only attribute characterizing the surface properties of nanomaterials that can be currently determined. The instruments are quite automatous, but their applications require precautions to achieve relevant measures. The value obtained is only valid within the framework of the experimental conditions used during determination.

\section{Analyzing attributes of nanomaterials using other methods.}

Lists of physical parameters recommended to be included to describe nanomaterials have been drawn in ISO/TR 13329:2012 (see table 1) and by different government agencies (FDA 2018 Rauscher et al., 2017, REACH legislation, 2006). In general, they all include the particle size, size distribution, aggregation and/or agglomeration state, a description of the particle shape. Depending on applications and chemical natures, other attributes are requested such as the crystallinity, the dispersibility, the dustiness and attributes related to the surface of the nanomaterials including the specific surface area (See ISO/TR 13329:2012 in table 1). Sometimes the porosity is included in the list of parameters to evaluate. The stability in terms of the maintenance of the colloidal stage is requested depending on the application envisaged. For nanomedicines the colloidal and chemical stability are important attributes to consider and more surface characteristics are requested. On a systematic basis, the surface charge needs to be provided. Recent results from the evaluation of nanomedicines have suggested that a more precise characterization of the surface attribute is needed to insure the reproducible in vivo fate of nanomaterials. Indeed, it was shown that small changes in subtle characteristics can generate a significant modification of the interactions between the nanomaterial and biological systems (Coty and Vauthier, 2018, Coty et al., 2017, Hamad et al., 2010) hence can modify the biodistribution and in turn greatly influence the activity and safety of the nanomedicine. Thus, parameters including the surface coverage, the topology, the configuration of the chains and the 
deformability of the surface were recently pointed out as possible characteristics to take into consideration as key surface attributes to insure in vivo safety and activity of nanomedicines.

As discussed earlier, little standardized methods were developed so far and only general attributes can be analyzed with automated methods applicable in routine. No method and easy to use instrument allowing convenient batch or lot quality evaluation are available to determine other attributes. Three situations can be distinguished. In the first case, a method exists but its application is tedious and have not been the subject of a standardization yet. This situation is found for the determination of the particle shape. In the second situation, methods applied for the characterization of other materials are used to characterize nanomaterials although measurement conditions are not ideal. The approach is generally used to characterize the porosity and of the crystallinity of nanomaterials. In the last situation, no easy methods can be applied to access other nanomaterial attributes. The characterization of the intimate structure of nanomaterials can be achieved using methods applied in research. The characterization of the surface of nanomaterials remains unsatisfied. Recently, a few initiatives have been taken to develop appropriate methods as explained bellow.

\subsection{Evaluating the shape of nanomaterials}

The shape of nanomaterial can be evaluated by appropriate microscopy methods. This can be done by the direct analysis of images produced during observations of samples by transmission electron microscopy (TEM), SEM or AFM. An aspect ratio was defined to quantify the shape of the observed nano-objects. It is deduced from the ratio between the longest and shortest axis measured in 2 directions on the obtained 2D images of nano-objects and differed from 1 when particles have a non-spherical or non-disc shape. To differentiate discs from spheres, 3D images are needed using SEM or AFM. The high of particles can be measured by AFM which allows measurements in the 3 dimensions of the space. As for measuring the size and size distribution of a sample, it is required to determine the aspect ratio of a large number of particles to perform 
relevant measurements. To the knowledge of the author, there is no image analyzers which can perform such an analysis which remains to be done manually. The general ISO standard describing the particle size analysis by image analysis methods can be used and that of the measurements of the particle size and shape distribution by SEM (See ISO/DIS19749 and ISO13322-1:2004 in table 1).

\subsection{Evaluating the porosity and crystallinity with general methods for the characterization of materials.}

In the absence of specific methods, the porosity and crystallinity of nanomaterials are often evaluated using existing methods applied for the characterization of non-nanomaterials. These methods are analyzing dried powders. Thus, prior performing the analysis, nanomaterials need to be dried. The obtained dry powder contains grains formed by an agglomerate of the nanoobjects composing the nanomaterial. Conditions for the evaluation of the porosity and crystallinity of the nanomaterials are not ideal as bias can be produced due to the arrangement of the nano-objects in the grains of the powder (Figure 2A). Pores can be formed between particles and may be encountered during the measurement of the porosity of the nanomaterial and crystallin arrangement of particles in the powder can produce diffraction peaks investigating the crystallinity of the nanomaterials (Figure 2B). Samples of materials are very different between those included nanomaterials and those found analyzing materials occurring as monolith. Cautious interpretation of data produced by these methods is then needed while they are applied to the characterization of nanomaterials. 

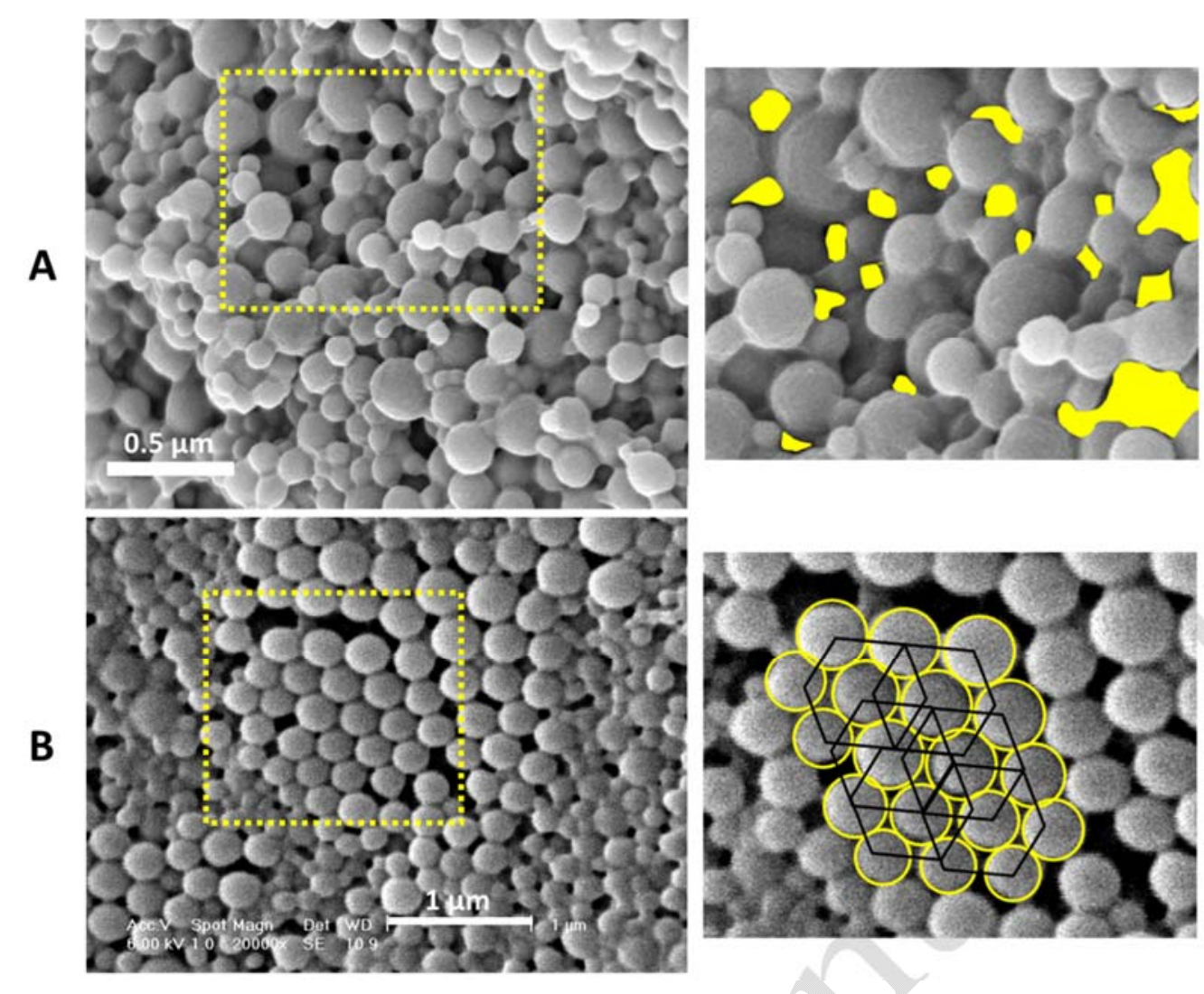

Figure 2: Possible bias evaluating nanoparticle attributes with methods not specifically developed for nanomaterials. (A) Determination of the porosity: Spaces between nanoparticles marked in yellow may represent a significative contribution to the porosity due to the small size of the nanomaterials contained in the dry powder of nanoparticles that is prepared to perform the analysis. (B) SEM picture of polymer nanoparticles showing a crystallin order in the arrangement of the nanoparticles. This type of crystallin arrangement of the nanoparticles within the dry sample prepared for X-Ray diffraction analysis can produce diffraction signals on X-ray diffractograms.

\subsection{Analysis of the intimate structure of nanomaterials}

The analysis of the intimate structure of nanomaterials need tremendous means implemented in synchrotrons and neutron scattering facilities. $\mathrm{X}$ rays and neutron scattering methods are suitable to decipher the arrangement of components within the structures of nano-objects (Vauthier et al., 2009, Lepeltier et al., 2013, Luo et al., 2018). In the nanomedicine domain, 
having access of these information may be useful to understand mechanisms of drug releasing property of the nanomaterial composing the pharmaceutical product. High resolution cryotransmission electron microscopies (cryo-TEM) would also be suitable techniques, but it has not been so much applied on the characterization of nanomaterials so far (Moradi et al., 2018, Angelov et al., 2015, Helvig et al., 2015). Cryo-TEM techniques are quite young methods and were initially developed for the analysis of biological materials including biomolecules and viruses using the high-resolution mode (Hong Zhou et al., 2011, Cressey et al., 2017, Shen, 2018). Currently, improvement of techniques continues and their application to the characterization of nanomaterial follows the evolution. Analysis of the intimate structure of nanomaterials remains an obstacle requiring rare instruments and the high expertise of season scientists.

\subsection{Thorough analysis of nanomaterial surface attributes}

Thorough analysis of nanomaterial surface remains challenging while surface properties beyond surface charge are often key parameters for the functionality of the particles (Baer et al., 2013). For instance, in the case of nanomedicines, attributes describing their surface characteristics are other parameters which play a major role to achieve the desired activity and safety of use. They control the biodistribution, cell internalization and interactions with biological molecules such as proteins in biological fluids and glycoproteins of the mucus on mucosa. Nanomedicines are nanomaterials that are to be introduced in the body intentionally. Establishing the link between surface attribute and biodistribution and activity is important to insure the quality and safety of the pharmaceutical product. With other manufactured nanomaterials which may be introduced in the body accidently, knowing such link would be worth to anticipate possible hazard. Currently the only "easy" way assessing surface property

of nanomaterial consists in evaluating the zeta potential (see section 3.3 in this chapter). The atomic composition of the surface can be analyzed by X-ray photo-electron spectroscopy 
(XPS). This method requires a specific instrumentation and a high level of expertise for the interpretation of the data while commonly applied in material science. The lateral resolution of the method is of the order of the micrometer and the depth of analysis is of a few nanometers (1 to 3) on metal surfaces but considering polymers, the depth of analysis can reach 6 to $7 \mathrm{~nm}$ [Kovac, 2011, Chan and Weng, 2016]. Spectra obtained from the analysis of the surface of materials composed of polymers may include signals from chemicals composing the underlying surface. The method was applied to analyze surface composition of polymer nanoparticles composing nanomedicines (Labarre et al., 2005, Layre et al., 2006, Baer et al., 2013). More specifically, it was used to demonstrate the presence of poly(ethylene glycol) or its absence on nanoparticle surfaces (See for instance Peracchia et al., 1998). This polymer was added on the surface of nanomedicines to reduce interactions with blood proteins that extend the time of residence of the nanoparticles in the blood stream allowing to modulate the in vivo fate of the nanoparticles administered by the intravenous route [Lakkireddy and Bazile, 2016, Suk et aL, 2016). Besides surface composition, the activity of a nanomedicine also depends on the fine tune of the density, distribution and conformation of the hydrophilic polymers added on the nanoparticle surface to control their interactions with biological systems hence the activity. Additionally, these characteristics influence the safety as they control the reactivity of undesired biochemical reactions as well as the organs in which nanomedicines accumulate in vivo. Evaluating of such subtle surface attributes of nanomedicines requires methods performing analysis at the molecular level. Such methods are currently missing. Existing methods analyze biological activities produced by the response of a biological system confronted to nanomaterials. For instance, they evaluate the response of the complement system which is a biochemical cascade of the innate immune system found in the plasma (NIH-NCL Assay Cascade, Dobrovolskaia et al., 2016). Others are based on the evaluation of the cytotoxicity of nanomaterials on define cell lines. Without possibility to characterize better surface attributes 
of nanomaterials, no correlation can be drawn between nanoparticle characteristics and the induced desired or unwanted biological responses. No progress in the understanding of this relation would be possible hampering rational design of nanomedicines and anticipation of eventual hazards from their physical attributes. Very recently, approaches were suggested based on the evaluation of the accessibility of the nanoparticle surface to proteins. In these approaches' proteins are used as molecular probes allowing to explore the particle surface at a molecular scale. The nature of the molecular probes is consistent with components of biological fluids which interact with nanomaterials entering the body. In one of the proposed methods, fluorescent proteins were used to assess the amount of the nanomaterial surface accessible for protein adsorption (Nasir et al., 2015). Another method using native proteins of different molecular weight could separate a series of nanoparticles in two groups consistently with steric hindrance properties of the surface coverage expected for the different nanoparticles included in the study. The observed responses resulted from the combined effect of the density of coverage and conformation of polysaccharide chains grafted on the nanoparticle surface (Coty et al., 2018). Both methods were conceived to be implemented for a routine application in quality control. More works is now requested to test the sensitivity of these methods considering more nanomaterials and to investigate whether the obtained data are relevant enough to insure the quality of nanomedicines based on the measured parameter. A difficulty to perform such evaluation is the absence of standard and/or reference materials. Besides, there is still no method to evaluate the topology and the deformability of nanoparticle surfaces while these attributes have been identified as other critical attributes for the activity and safety of nanomedicines.

\section{Conclusion}

Analyzing nanomaterials remains a challenge due to the small size of nano-objects that composed such materials. The characterization and analysis of physical attributes of nanomaterials requires specific methods. At present, only very few general attributes can be 
analyzed by standardized methods. Affordable automated instruments can be used to evaluate the size, polydispersity and surface charge choosing suitable method depending on the sample characteristics such as its polydispersity and chemical composition. A tremendous need of accessible measurement methods analyzing attributes that define activity and safety of nanomaterials was pointed out. Issues are several including the lack of methods applicable in quality control, the lack of standard and reference materials to qualify measurement protocols and instruments, the lack of standardized methods and the lack of methods. Works are on the way to fill the gaps and resolve the analytical needs of products composed of nanomaterials to insure their quality and safety.

\section{References}

Anchordoquy TJ, Barenholz Y, Boraschi D, et al: Mechanisms and Barriers in Cancer Nanomedicine: Addressing Challenges, Looking for Solutions. ACS Nano 11:12-18, 2017.

Anderson W, Kozak D, Coleman VA, et al: A comparative study of submicron particle sizing platforms: accuracy, precision and resolution analysis of polydisperse particle size distributions. J Colloid Interface Sci 405:322-330, 2013.

Angelov B, Angelova A, Drechsler M, et al: Identification of large channels in cationic PEGylated cubosome nanoparticles by synchrotron radiation SAXS and Cryo-TEM imaging. Soft Matter 11:3686-3692, 2015.

Baer DR, Engelhard MH, Johnson GE: Surface characterization of nanomaterials and nanoparticles: Important needs and challenging opportunities. J Vac Sci Technol A 31:50820, 2013

Bremer-Hoffmann S., Halamoda-Kenzaoui B, Borgos SE: Identification of regulatory needs for nanomedicines. J Interdis Nanomed 3:4-15, 2018. 
Chan CM, Weng LT: Surface Characterization of Polymer Blends by XPS and ToF-SIMS. Materials (Basel). 9(8). pii: E655, 2016.

Chaurasia SS, Lim RR, Lakshminarayanan R: Nanomedicine Approaches for Corneal Diseases. J. Funct. Biomater. 6:277-298, 2015.

Clogston JD, Crist RM, McNeil SE: Physicochemical characterization of polymer nanoparticles: challenges and present limitations. In Vauthier $\mathrm{C}$, Ponchel $\mathrm{G}$, editors: Polymer nanoparticles for nanomedicines. Cham, Switzerland, 2016, Springer International Publishing, pp 187-203.

Coty J.B, Vauthier C: Characterization of nanomedicines: a reflection on a field under construction needed for clinical translation success. J. Control. Rel 275:254-268, 2018.

Coty J.B., Eleamen Oliviera E., Vauthier C: Tuning complement activation and pathway through controlled molecular architecture of dextran chains in nanoparticle corona. Int $J$ Pharm 532:769-778, 2017.

Cressey D, Callaway E. Cryo-electron microscopy wins chemistry Nobel: Jacques Dubochet, Joachim Frank and Richard Henderson share the prize for developing a technique to image biomolecules. Nature. 550: 167, 2017.

Christ RM, Hall Grossman J, Patri AK: Common Pitfalls in Nanotechnology: Lessons Learned from NCI's Nanotechnology Characterization Laboratory. Integr Biol (Camb). 5:10.1039/c2ib20117h, 2013.

Coty JB, Varenne F, Benmalek A: Characterization of nanomedicines' surface coverage using molecular probes and capillary electrophoresis. Eur J Pharm Biopharm 130:48-58, 2018. 
Coty JB, Vauthier C: Characterization of nanomedicines: A reflection on a field under construction needed for clinical translation success. J Control Release. 275:254-268, 2018.

Coty JB, Eleamen Oliveira E, Vauthier C: Tuning complement activation and pathway through controlled molecular architecture of dextran chains in nanoparticle corona. Int J Pharm $532: 769-778,2017$.

Cruz CN, Tyner KM, Velazquez L: CDER risk assessment exercise to evaluate potential risks from the use of nanomaterials in drug products. AAPS $J$ 15:623-628, 2013.

Dobrovolskaia MA: Pre-clinical immunotoxicity studies of nanotechnology-formulated drugs: Challenges, considerations and strategy. J Control Release. 220(Pt B):571-583, 2015.

Dobrovolskaia MA, Shurin M, Shvedova AA : Current understanding of interactions between nanoparticles and the immune system. Toxicol Appl Pharmacol. 299:78-89, 2016.

EU-NCL Assay Cascade (web site) www.euncl.eu/about-us/assay-cascade/. Accessed January $16,2019$.

EU-NCL (web site) www.euncl.eu/about-us/overview/. Accessed January 16, 2019.

FDA 2018 Nanotechnology guidance documents updated 23 March 2018 (web site) www.fda.gov/ScienceResearch/SpecialTopics/Nanotechnology/ucm602536.htm and $\begin{array}{llllll}\text { Nanotechnology fact } & \text { sheets updated } 23 & \text { March }\end{array}$ www.fda.gov/ScienceResearch/SpecialTopics/Nanotechnology/ucm402230.htm. Accessed January 27, 2019.

Filipe V, Hawe A, Jiskoot W: Critical evaluation of Nanoparticle Tracking Analysis (NTA) by NanoSight for the measurement of nanoparticles and protein aggregates. Pharm Res $27: 796-810,2010$. 
Gioria S, Caputo F, Urbán P: Are existing standard methods suitable for the evaluation of nanomedicines: some case studies. Nanomedicine (Lond). 13:539-554, 2018.

GUM 1995. Guide to the expression of uncertainty in measurement, JCGM 100:2008 GUM1995 with minor corrections. (web site) www.bipm.org/utils/common/documents/jcgm/JCGM 1002008 E.pdf. Accessed January 27, 2019.

Hamad I, Al-Hanbali O, Hunter AC: Distinct polymer architecture mediates switching of complement activation pathways at the nanosphere-serum interface: implications for stealth nanoparticle engineering. ACS Nano 4:6629-6638, 2010.

Helvig S, Azmi IDM, Moghimi SM: Recent Advances in Cryo-TEM Imaging of Soft Lipid Nanoparticles. AIMS Biophys 2:116-130, 2015.

Hong Zhou Z: Atomic resolution cryo-electron microscopy of macromolecular complexes. Adv Protein Chem Struct Biol 82:1-35, 2011.

Hussain CM, Editor: Nanomaterials in chromatography: current trends in chromatographic research technology and techniques, 2018, Elsevier. https://doi.org/10.1016/C2016-004157-8.

ICH Harmonized Tripartite Guideline, Validation of analytical procedures: Text and Methodology Q2(R1), Current Step 4 version, Parent Guideline dated 27 October 1994, (Complementary Guideline on Methodology dated 6November 1996 incorporated in November 2005). (web site) www.ich.org/products/guidelines/quality/article/qualityguidelines.html. Accessed January 27, 2019.

ISO (web site) https://www.iso.org/home.html. Accessed January 21, 2019. 
Juliano R: The delivery of therapeutic oligonucleotides. Nucleic Acids Res 44:6518-6548, 2016.

Kamaly N, Xiao Z, Valencia PM: Targeted polymeric therapeutic nanoparticles: design, development and clinical translation. Chem Soc Rev 41:2971-3010, 2012.

Kovac J: Surface characterization of polymers by XPS and SIMS techniques. Materials and technology 3, 191-197, 2011.

Labarre D, Vauthier C, Chauvierre C: Interactions of blood proteins with poly(isobutylcyanoacrylate) nanoparticles decorated with a polysaccharidic brush. Biomaterials 26:5075-5084, 2005.

Lakkireddy HR, Bazile D: Building the design, translation and development principles of polymeric nanomedicines using the case of clinically advanced poly(lactide(glycolide))poly(ethylene glycol) nanotechnology as a model: An industrial viewpoint. Adv Drug Deliv Rev 107:289-332, 2016.

Lakkireddy HR, Urmann M, Besenius M: Oral delivery of diabetes peptides - Comparing standard formulations incorporating functional excipients and nanotechnologies in the translational context. Adv Drug Deliv Rev 106(Pt B):196-222, 2016.

Lamch L, Pucek A, Kulbacka J, et al: Recent progress in the engineering of multifunctional colloidal nanoparticles for enhanced photodynamic therapy and bioimaging. Adv Colloid Interface Sci. 261:62-81, 2018.

Langevin D, Lozano O, Salvati A: Inter-laboratory comparison of nanoparticle size measurements using dynamic light scattering and differential centrifugal sedimentation. NanoImpact 10, 97-107, 2018 a. 
Langevin D, Raspaud E, Mariot S: Towards reproducible measurement of nanoparticle size using dynamic light scattering: Important controls and considerations. NanoImpact 10, 161-167, $2018 b$.

Layre A, Couvreur P, Chacun H et al: Novel composite core-shell nanoparticles as busulfan carriers. J Control Release 111:271-280, 2006.

Lepeltier E, Bourgaux C, Rosilio V et al: Self-assembly of squalene-based nucleolipids: relating the chemical structure of the bioconjugates to the architecture of the nanoparticles. Langmuir 29:14795-14803, 2013.

Luo Z, Marson D, Ong QK: et al : Quantitative 3D determination of self-assembled structures on nanoparticles using small angle neutron scattering. Nat Commun 9:1343, 2018.

Mansfield E, Kaiser DL, Fujita D et al. editors: Metrology and Standardization for Nanotechnology: Protocols and Industrial Innovations. Weinheim, Germany, 2017, Wiley-VCH Verlag GmbH \& Co. KGaA, ISBN: 978-3-527-34039. 626 pages.

Moradi MA, Bomans PHH, Jackso AW, et al.: A quantitative cryoTEM study on crosslinked nanocapsule morphology in RAFT-based vesicle polymerization. Eur Polym J 108:329$336,2018$.

Mura S, Couvreur P, editors: Nanoteranostic for personalized medicine, LOCATION, 2016, World Scientific. https://doi.org/10.1142/9741.

Nanomaterial definitions: ec.europa.eu/environment/chemicals/nanotech/faq/definition_en.htm. (web site) Accessed January 24，2019， www.fda.gov/RegulatoryInformation/Guidances/ucm257698.htm, (web site) Accessed January 24, 2019, ISO/TR 18401:2017(en) Nanotechnologies — Plain language explanation of selected terms from the ISO/IEC 80004 series (web site) 
www.iso.org/obp/ui/\#iso:std:iso:tr:18401:ed-1:v1:en. (web site) Accessed January 24, 2019.

Nasir I, Fatih W, Svensson A, et al: High Throughput Screening Method to Explore Protein Interactions with Nanoparticles. PLoS One 10:e0136687, 2015.

NIH-NCL (web site). ncl.cancer.gov/. Accessed January 16, 2019.

NIH-NCL Assay cascade. (web site) ncl.cancer.gov/resources/assay-cascade-protocols. Accessed January, 162019.

Peracchia MT, Vauthier C, Desmaële D, et al:. Pegylated nanoparticles from a novel methoxypolyethylene glycol cyanoacrylate-hexadecyl cyanoacrylate amphiphilic copolymer. Pharm Res. 15:550-556, 1998.

Pelaz B., C. Alexiou, R. Alvarez-Puebla, et al: Diverse Applications of Nanomedicine. ACS Nano. 11:2313-2381,2017.

Petersen EJ, Henry TB, Zhao J, et al: Identification and avoidance of potential artifacts and misinterpretations in nanomaterial ecotoxicity measurements. Environ Sci Technol 48:4226-4246, 2014.

Rauscher H, Rasmussen K, Sokull-Klüugen B. Regulatory aspects of nanomaterials in the EU. Chem Ing Tech 89:224-231, 2017.

REACH legislation from 18 December 2006, Regulation (EC) No 1907/2006 (web site) echa.europa.eu/regulations/reach/legislation. Accessed January 16, 2019.

Shen PS. The 2017 Nobel Prize in Chemistry: cryo-EM comes of age. Anal Bioanal Chem 410:2053-2057, 2018 
Siegrist S, Cörek E, Detampel P et al: Preclinical hazard evaluation strategy for nanomedicines. Nanotoxicology. 5:1-27, 2018.

Singer A, Markoutsa E, Limayem A, et al: Nanobiotechnology Medical Applications: Overcoming Challenges Through Innovation. The EuroBiotech Journal 2:146-160, 2018.

Suk JS, Xu Q, Kim N, et al: PEGylation as a strategy for improving nanoparticle-based drug and gene delivery. Adv Drug Deliv Rev 99:28-51, 2016.

Thirumavalavan M, Settu K, Lee JF: A Short Review on Applications of Nanomaterials in Biotechnology and Pharmacology. Current Bionanotechnology 2:116-121, 2016.

Ventola CL: Progress in Nanomedicine: Approved and Investigational Nanodrugs. P T 42:742$755,2017$.

Varenne F, Botton J, Merlet C, et al: Standardization and validation of a protocol of size measurements by dynamic light scattering for monodispersed stable nanomaterial characterization. Colloid Surf A. 486:124-138, 2015 a.

Varenne F, Botton J, Merlet C, et al: Standardization and validation of a protocol of zeta potential measurements by electrophoretic light scattering for nanomaterial characterization, Colloid. Surface. A 486:218-231, 2015b. Erratum to [Colloid. Surface. A 486 (2015b) 218-231] Colloid. Surface. A 498:283-284, 2016.

Varenne F, Botton J, Merlet M, et al: Size of monodispersed nanomaterials evaluated by dynamic light scattering: Protocol validated for measurements of 60 and $203 \mathrm{~nm}$ diameter nanomaterials is now extended to 100 and $400 \mathrm{~nm}$, Int J Pharm 515:245-253, 2016a. 
Varenne F, Makky A, Gaucher-Delmas M, et al: Multimodal Dispersion of Nanoparticles: A Comprehensive Evaluation of Size Distribution with 9 Size Measurement Methods. Pharm Res 33:1220-1234, 2016b.

Varenne F, Rustique E, Botton J, et al: Towards quality assessed characterization of nanomaterial: Transfer of validated protocols for size measurement by dynamic light scattering and evaluation of zeta potential by electrophoretic light scattering, Int J Pharm 528:299-311, 2017.

Varenne F, Hillaireau H, Bataille J, et al: Application of Validated Protocols to Characterize Size and Zeta Potential of Dispersed Materials using Light Scattering Methods, Colloid. Surface. A In Press Doi.org/10.1016/j.colsurfa.2018.09.006.

Vauthier C, Lindner P, Cabane B: Configuration of bovine serum albumin adsorbed on polymer particles with grafted dextran corona. Colloids Surf B Biointerfaces 69:207-215, 2009.

Zhang XQ, Xu X, Bertrand N, et al: Interactions of nanomaterials and biological systems: Implications to personalized nanomedicine. Adv Drug Deliv Rev 64:1363-1384, 2012. 